\title{
A note on Lyapunov exponents of deterministic strongly mixing potentials
}

\author{
Jean Bourgain and Eric Bourgain-Chang
}

\begin{abstract}
In this Note, we consider 1D lattice Schrödinger operators with deterministic strongly mixing potentials as studied in [3] and [2] with very small coupling. We describe a scheme to establish positivity of the Lyapunov exponent from a statement at some fixed scale. The required input may then be derived from Furstenberg theory, if the underlying dynamics are sufficiently mixing, or verified directly by numerical means.
\end{abstract}

Mathematics Subject Classification (2010). 39A70, 47B39, 47N50, 82B44.

Keywords. Lyapounov exponent,Schrödinger operator,transfer matrix.

\section{Summary}

Consider the two following model cases of Schrodinger operators on $\mathbb{Z}$

$$
H_{x}=\Delta+V_{x} \quad \text { with } V_{x}(j)=\lambda f\left(K^{j} x\right), x \in \mathbb{T}, K \in \mathbb{Z}_{+}, K \geq 2
$$

and

$$
H_{x}=\Delta+V_{x} \quad \text { with } V_{x}(j)=\lambda f\left(A^{j} x\right), x \in \mathbb{T}^{2}, A \in \mathrm{SL}_{2}(\mathbb{Z}) \text { hyperbolic. }
$$

Here $f$ is a non-constant function that we assume at least $C^{1}$.

For small coupling $\lambda \neq 0$, positivity of the Lyapunov exponents was established in [3] using the Figotin-Pastur perturbative method. This analysis was pursued in [2] where we proved furthermore that the Integrated Density of States (IDS) is Hölder regular and $H_{x}$ satisfies Anderson localization for almost every $x$.

At the other end, if we assume for instance $f$ a non-constant trigonometric polynomial and $|\lambda|>\lambda_{f}$, M. Herman subharmonicity technique applies to prove positivity of the exponents (and hence Anderson localization using the arguments from [2]). 
These seemed to be the only available strategies, which do not cover intermediate ranges of $\lambda$ (note also that Herman's method is quite restrictive for the function $f$ ).

The purpose of this Note is to show how to prove the above properties (under mild assumptions on $f$ ) and arbitrary $\lambda \neq 0$, provided the transformation is assumed 'sufficiently mixing'. More precisely, in (1.1) we assume $K$ large enough and in (1.2), that $A$ is sufficiently expanding, depending on the oscillation of $f$ and $\|f\|_{C^{1}}$. Eventually, positivity of the Lyapunov exponents is then derived from Furstenberg's theorem on random matrix products.

Precise statements appear in Theorems 1 and 2 below.

Roughly speaking, our approach relies on a finite scale statement. This input is derived from large deviation principles in Furstenberg's theory, arguing that the cocycles at some fixed finite scale behave like in the random case (for an appropriate assumption on the model). Rather than invoking random matrix product theory, the required finite scale information may also be checked numerically, as we will illustrate in some simple cases. We also believe that such "finitary approach" to problems of positivity of Lyapunov exponents could be useful in other situations.

\section{Schrodinger cocycles}

Let $(\Omega, \mu, T)$ be a dynamical system, $\Omega=T^{k}$ a torus, $\mu$ Lebesque. This setting covers our examples.

Let $f$ be a $C^{1}$ function on $\Omega$.

Define

$$
\begin{aligned}
& M_{N}(x) \\
& \quad=\left(\begin{array}{cc}
E-f\left(T^{N-1} x\right) & -1 \\
1 & 0
\end{array}\right)\left(\begin{array}{cc}
E-f\left(T^{N-2} x\right) & -1 \\
1 & 0
\end{array}\right) \cdots\left(\begin{array}{cc}
E-f(x) & -1 \\
1 & 0
\end{array}\right) .
\end{aligned}
$$

Let $N_{0}$ be a fixed large scale and write

$$
M_{N N_{0}}(x)=M_{N_{0}}\left(T^{(N-1) N_{0}} x\right) M_{N_{0}}\left(T^{(N-2) N_{0}} x\right) \ldots M_{N_{0}}(x) .
$$

Next, we perform a translation of $x$ by vectors $y \in \Omega_{N_{0}, N} \subset \Omega$ (where $\Omega_{N_{0}, N}$ may be finite or infinite) and equipped with a normalized measure $A_{y} v$. Using again the 
cocycle property

$$
\begin{aligned}
\log \| & M_{N N_{0}}(x+y) v \| \\
= & \log \left\|M_{N_{0}}\left(T^{(N-1) N_{0}}(x+y)\right)\left(\frac{M_{(N-1) N_{0}}(x) v}{\left\|M_{(N-1) N_{0}}(x) v\right\|}\right)\right\| \\
+ & \log \left\|M_{(N-1) N_{0}}(x) v\right\| \\
+ & {\left[\log \left\|M_{N N_{0}}(x+y) v\right\|\right.} \\
& \left.\quad-\log \left\|M_{N_{0}}\left(T^{(N-1) N_{0}}(x+y)\right) M_{(N-1) N_{0}}(x) v\right\|\right] .
\end{aligned}
$$

Integrating, this gives

$$
\begin{aligned}
& \int \log \left\|M_{N N_{0}}(x) v\right\| d x \geq \\
& \int\left\{A_{y} \log \left\|M_{N_{0}}\left(T^{(N-1) N_{0}}(x+y)\right)\left(\frac{M_{(N-1) N_{0}}(x) v}{\left\|M_{(N-1) N_{0}}(x) v\right\|}\right)\right\|\right\} d x \\
& +\int_{-(2.5)} \log \left\|M_{(N-1) N_{0}}(x) v\right\| d x
\end{aligned}
$$

with (2.5) an upperbound on $|(2.3)|$ for $x \in \Omega$ and $y \in \Omega_{N_{0}, N}$.

Obviously (2.4) is at least

$$
\min _{N} \min _{x \in \Omega,|w|=1} A v\left\{\log \left\|M_{N_{0}}\left(T^{(N-1) N_{0}}(x+y)\right) w\right\|\right\} .
$$

Our goal is then to ensure positivity of (2.6), more precisely,

$$
\text { (2.6) }>(2.5) \text {. }
$$

This process may then be iterated, leading to positivity of the Lyapunov exponent

$$
L(E)=\lim _{N \rightarrow \infty} \frac{1}{N} \int_{\Omega} \log \left\|M_{N}(E, x)\right\| d x .
$$

The scale $N_{0}$ is chosen sufficiently large, depending on the dynamics and $f$. The basic idea is to obtain an image measure on $\Omega^{N_{0}}$ under the map

$$
y \longmapsto\left(T^{(N-1) N_{0}}(x+y), T^{(N-1) N_{0}+1}(x+y), \ldots, T^{N N_{0}-1}(x+y)\right)
$$

which is close to the uniform measure on $\Omega^{N_{0}}$ (in the weak sense, depending on $f$ ). This will require sufficiently strong mixing properties for the transformation $T$. Eventually (assuming $f$ non-constant of course), our goal is to exploit 
the theory of random matrix products, cf. [1]. This theory indeed asserts that by taking $N_{0}$ large enough,

$$
\min _{|w|=1} \int_{\Omega^{N_{0}}} \log \left\|\prod_{j=0}^{N_{0}-1}\left(\begin{array}{cc}
E-f\left(x_{j}\right) & -1 \\
1 & 0
\end{array}\right) w\right\| d x_{0} \cdots d x_{N_{0}-1}>c N_{0}
$$

for $c \approx L(E)>0$ and (2.8) moreover valid for all $E$ in a given bounded energy interval (note that for large $E$, clearly $L(E) \sim \log |E|$ ).

It remains then to bound (2.5), which is an issue of cocycle perturbation and will typically depend on expansion properties of $T$. Some basic estimates will be presented in the next section.

There is the following variant of the above construction. Taking $v \in \mathbb{R}^{2}$, $|v|=1$, one may write (denoting $M_{N}^{*}$ the adjoint of $M_{N}$ )

$$
\begin{aligned}
& \log \| M_{N N_{0}}(x+y) \\
& \geq \log \left\|M_{N N_{0}}^{*}(x+y) v\right\| \\
& \geq \log \left\|M_{N_{0}}^{*}(x+y) \frac{M_{(N-1) N_{0}}^{*}\left(T^{N_{0}} x\right) v}{\left\|M_{(N-1) N_{0}}^{*}\left(T^{N_{0}} x\right) v\right\|}\right\| \\
& \quad+\log \left\|M_{(N-1) N_{0}}^{*}\left(T^{N_{0}} x\right) v\right\| \\
& \quad-\max _{|v|=1, y \in \Omega_{N_{0}, N}} \mid \log \left\|M_{N N_{0}}^{*}(x+y) v\right\| \\
& \quad-\log \left\|M_{N_{0}}^{*}(x+y) M_{(N-1) N_{0}}^{*}\left(T^{N_{0}} x\right) v\right\| \mid .
\end{aligned}
$$

Let then $v=v_{x}$ such that $\left\|M_{(N-1) N_{0}}^{*}\left(T^{N_{0}} x\right) v_{x}\right\|=\left\|M_{(N-1) N_{0}}\left(T^{N_{0}} x\right)\right\|$. Hence

$$
(2.9)+(2.10)=\log \left\|M_{N_{0}}^{*}(x+y) w_{x}\right\|+\log \left\|M_{(N-1) N_{0}}\left(T^{N_{0}} x\right)\right\|
$$

for some unit vector $w_{x}$. Integration in $x \in \Omega, y \in \Omega_{N_{0}, N}$ gives

$$
\begin{aligned}
\int \log \left\|M_{N N_{0}}(x)\right\| d x> & \min _{x \in \Omega,|w|=1}\left\{A_{y} v \log \left\|M_{\left(N_{0}\right)}^{*}(x+y) w\right\|\right\} \\
& +\int \log \left\|M_{(N-1) N_{0}}(x)\right\| d x \\
& -(2.11)
\end{aligned}
$$

and we may iterate, provided (2.12) $-(2.11)>0$.

We will implement the above scheme in the following examples:

$$
\begin{gathered}
\Omega=\mathrm{T}, \quad T x=K x, \quad \text { with } K \geq 2 \text { an integer; } \\
\Omega=\mathbb{T}^{2}, \quad T x=A x, \quad \text { with } A \in \mathrm{SL}_{2}(\mathbb{Z}) \text { hyperbolic. }
\end{gathered}
$$




\section{Perturbation of cocycles}

Let

$$
\begin{aligned}
& A_{1}, \ldots, A_{N} \in \mathrm{SL}_{2}(\mathbb{R}), \quad\left\|A_{j}\right\|,\left\|A_{j}^{-1}\right\|<C_{1}, \\
& B_{1}, \ldots, B_{N} \in \mathrm{SL}_{2}(\mathbb{R}), \quad\left\|A_{j}-B_{j}\right\|<\varepsilon_{j}<1,
\end{aligned}
$$

and

$$
v \in \mathbb{R}^{2},\|v\|=1 \text {. }
$$

The purpose of what follows is to establish inequality (3.11) below.

Estimate

$$
\begin{aligned}
& \left\|A_{1} \ldots A_{N} v-B_{1} \ldots B_{N} v\right\| \\
& \quad \leq\left\|A_{1}-B_{1}\right\|\left\|A_{2} \ldots A_{N} v\right\|+\left\|B_{1} A_{2} \ldots A_{N} v-B_{1} \ldots B_{N} v\right\| \\
& \quad=(3.1)+(3.2) .
\end{aligned}
$$

Then

$$
\begin{gathered}
(3.1) \leq \varepsilon_{1}\left\|A_{2} \cdots A_{N} v\right\| \\
\leq \varepsilon\left\|A_{1}^{-1}\right\|\left\|A_{1} \cdots A_{N} v\right\| \\
<\varepsilon_{1} C_{1}\left\|A_{1} \ldots A_{N} v\right\|, \\
(3.2) \leq\left\|B_{1}\right\|\left\|A_{2}-B_{2}\right\|\left\|A_{3} \ldots A_{N} v\right\| \\
+\left\|B_{1} B_{2} A_{3} \ldots A_{N} v-B_{1} \ldots B_{N} v\right\| \\
=(3.3)+(3.4), \\
(3.3) \leq\left(C_{1}+\varepsilon_{1}\right) \varepsilon_{2}\left\|A_{3} \ldots A_{N} v\right\| \\
\leq\left(C_{1}+1\right) \varepsilon_{2}\left\|A_{2}^{-1}\right\|\left\|A_{1}^{-1}\right\|\left\|A_{1} A_{2} \ldots A_{N} v\right\| \\
<\varepsilon_{2}\left(1+C_{1}\right) C_{1}^{2}\left\|A_{1} \ldots A_{N} v\right\|, \\
(3.4) \leq\left\|B_{1}\right\|\left\|B_{2}\right\|\left\|A_{3}-B_{3}\right\|\left\|A_{4} \cdots A_{N} v\right\| \\
+\left\|B_{1} B_{2} B_{3} A_{4} \ldots A_{N} v-B_{1} \ldots B_{N} v\right\| \\
=(3.5)+(3.6),
\end{gathered}
$$




$$
\begin{aligned}
(3.5) & <\left(1+C_{1}\right)^{2} \varepsilon_{3}\left\|A_{3}^{-1}\right\|\left\|A_{2}^{-1}\right\|\left\|A_{1}^{-1}\right\|\left\|A_{1} A_{2} \ldots A_{N} v\right\| \\
& <\varepsilon_{3}\left(1+C_{1}\right)^{2} C_{1}^{3}\left\|A_{1} \ldots A_{N} v\right\|,
\end{aligned}
$$

etc.

Hence

$$
\left\|A_{1} \ldots A_{N} v-B_{1} \ldots B_{N} v\right\|<\left[\sum_{j \geq 1} \varepsilon_{j}\left(1+C_{1}\right)^{2 j-1}\right]\left\|A_{1} \ldots A_{N} v\right\|
$$

and also

$$
\left\|\frac{A_{1} \ldots A_{N} v}{\left\|A_{1} \ldots A_{N} v\right\|}-\frac{B_{1} \ldots B_{N} v}{\left\|B_{1} \ldots B_{N} v\right\|}\right\|<2 \sum_{j \geq 1} \varepsilon_{j}\left(1+C_{1}\right)^{2 j-1} .
$$

Write a telescopic sum

$$
\begin{aligned}
\log \left\|A_{1} \ldots A_{N} v\right\| & \\
= & \log \left\|A_{1}\left(\frac{A_{2} \ldots A_{N} v}{\left\|A_{2} \ldots A_{N} v\right\|}\right)\right\|+\log \left\|A_{2}\left(\frac{A_{3} \ldots A_{N} v}{\left\|A_{3} \ldots A_{N} v\right\|}\right)\right\| \\
& +\cdots+\log \left\|A_{j}\left(\frac{A_{j+1} \ldots A_{N} v}{\left\|A_{j+1} \ldots A_{N} v\right\|}\right)\right\|+\ldots
\end{aligned}
$$

By (3.8),

$$
\begin{aligned}
& \left\|A_{j}\left(\frac{A_{j+1} \ldots A_{N} v}{\left\|A_{j+1} \ldots A_{N} v\right\|}\right)-B_{j}\left(\frac{B_{j+1} \ldots B_{n} v}{\left\|B_{j+1} \ldots B_{N} v\right\|}\right)\right\| \\
& \quad<\varepsilon_{j}+2 C_{1} \sum_{j^{\prime} \geq j+1} \varepsilon_{j^{\prime}}\left(1+C_{1}\right)^{2 j^{\prime}-1},
\end{aligned}
$$

and therefore, since $\left\|B_{j} w\right\| \geq\left\|B_{j}^{-1}\right\|^{-1}>\frac{1}{C_{1}}$ for $|w|=1$,

$$
|| \frac{A_{j}\left(\frac{A_{j+1} \ldots A_{N} v}{\left\|A_{j+1} \cdots A_{N} v\right\|}\right) \|}{\left\|B_{j}\left(\frac{B_{j+1} \ldots B_{N} v}{\left\|B_{j+1} \ldots B_{N} v\right\|}\right)\right\|}-1 \mid<C_{1} \varepsilon_{j}+2 C_{1}^{2} \sum_{j^{\prime} \geq j+1} \varepsilon_{j^{\prime}}\left(1+C_{1}\right)^{2 j^{\prime}-1} .
$$

Hence, using the inequality $|\log (1+x)|<2|x|$ for $|x|<\frac{1}{2}$, it follows that

$$
\begin{aligned}
& \left|\log \left\|A_{j}\left(\frac{A_{j+1} \ldots A_{N} v}{\left\|A_{j+1} \ldots A_{N} v\right\|}\right)\right\|-\log \left\|B_{j}\left(\frac{B_{j+1} \ldots B_{N} v}{\left\|B_{j+1} \ldots B_{N} v\right\|}\right)\right\|\right| \\
& \quad<2 C_{1} \varepsilon_{j}+4 C_{1}^{2} \sum_{j^{\prime} \geq j+1} \varepsilon_{j^{\prime}}\left(1+C_{1}\right)^{2 j^{\prime}-1},
\end{aligned}
$$


provided

$$
C_{1}^{2} \sum_{j \geq 1} \varepsilon_{j}\left(1+C_{1}\right)^{2 j-1}<\frac{1}{5}
$$

Assuming (3.10), it follows that

$$
\left|\log \left\|A_{1} \ldots A_{N} v\right\|-\log \left\|B_{1} \ldots B_{N} v\right\|\right|<6 C_{1}^{2} \sum_{j \geq 1} j \varepsilon_{j}\left(1+C_{1}\right)^{2 j-1} .
$$

\section{Map $x \mapsto K x$ on $\mathbb{T}$}

Let $\Omega=\mathbb{T}, K \in \mathbb{Z}_{+}, K \geq 2$ and $f \in C^{1}(\mathbb{T})$. We apply the second procedure discussed in Section 2.

Fix $N_{0}$ and define

$$
\Omega_{N_{0}, N}=\Omega_{N_{0}}=\left\{\frac{j}{K^{N_{0}}} ; j=0,1, \ldots, K^{N_{0}}-1\right\},
$$

noting that

$$
T^{N_{0}}(x+y) \equiv T^{N_{0}}(x \bmod 1) \quad \text { for all } x \in \mathbb{T}, y \in \Omega_{N_{0}} .
$$

Hence $(2.11)=0$ and (2.12) equals

$$
\begin{aligned}
\min _{x,|w|=1}\left\{K^{-N_{0}} \sum_{\alpha=0}^{K^{N_{0}-1} \log }\right. & \left(\begin{array}{cc}
E-f\left(x+K^{-N_{0}} \alpha\right) & 1 \\
-1 & 0
\end{array}\right) \\
& \left(\begin{array}{cc}
E-f\left(K x+K^{-N_{0}+1} \alpha\right) & 1 \\
-1 & 0
\end{array}\right) \cdots \\
& \left.\left(\begin{array}{cc}
E-f\left(K^{N_{0}-1} x+\frac{\alpha}{K}\right) & 1 \\
1 & 0
\end{array}\right)\left(\begin{array}{l}
w_{1} \\
w_{2}
\end{array}\right) \|\right\} .
\end{aligned}
$$

It remains to analyze the map

$$
\varphi=\varphi_{x}: \mathbb{Z} / K^{N_{0}} \mathbb{Z} \longrightarrow \mathbb{T}^{N_{0}}
$$

defined by

$$
\varphi(\alpha)=\left(x+\frac{\alpha}{K^{N_{0}}}, K x+\frac{\alpha}{K^{N_{0}-1}}, \ldots, K^{N_{0}-1} x+\frac{\alpha}{K}\right) .
$$

If we fix $N_{0}$ and take $K$ large enough, the image measure becomes weakly equidistributed (uniformly in $x$ ). One has indeed that for $\xi \in \mathbb{Z}^{N_{0}}, 0<|\xi|<K$,

$$
K^{-N_{0}}\left|\sum_{\alpha=0}^{K^{N_{0}-1}} e^{2 \pi i \xi . \varphi(\alpha)}\right|=K^{-N_{0}}\left|\sum_{\alpha=0}^{K^{N_{0}-1}} e^{2 \pi i \alpha\left(\frac{\xi_{0}}{K^{N}}+\frac{\xi_{1}}{K^{N}-1}+\cdots+\frac{\xi_{N_{0}-1}}{K}\right)}\right|=0
$$

implying the required equidistribution statement. 
In view of the discussion in Section 2, we proved the following result.

Theorem 1. Given $\kappa>0$ and $0<C<\infty$, there is $K_{0}$ such that if $K \in \mathbb{Z}_{+}$, $K>K_{0}$, and $f \in C^{1}(\mathrm{~T})$ is a real function satisfying

$$
\|f\|_{C^{1}}<C \text { and } \operatorname{osc}(f)>\kappa
$$

then the Schrodinger operator on $\mathbb{Z}_{+}$

$$
H_{x}=\Delta+V_{x}, \quad \text { with } V_{x}(j)=f\left(K^{j} x\right),
$$

has positive Lyapunov exponents for all energies.

Once the positivity of the Lyapunov exponents established, we may proceed further, following [2], and prove a large deviation inequality for the pointwise Lyapunov exponents

$$
L_{N}(E ; x)=\frac{1}{N} \log \left\|M_{N}(E ; x)\right\|
$$

This enables then to establish Hölder regularity of $L(E)$ and the IDS $N(E)$ (by Thouless formula) and also Anderson localization (see [2] for details).

Recall that the large deviation estimate required in this analysis is of the form

$$
\operatorname{mes}\left[x \in \mathbb{T} ;\left|L_{N}(E, x)-L_{N}(E)\right|>o(1) L_{N}(E)\right]<e^{-c N}
$$

where $L_{N}(E)=\int_{\mathbb{T}} L_{N}(E, x) d x$.

We indicate a proof of (4.5) by elaborating upon the above considerations.

Denote $\prod$ the product space $\prod_{\mathbb{Z}_{+}}\left\{0,1 \ldots, K^{N_{0}}-1\right\}$ equipped with normalized product measure. Given $\alpha=\left(\alpha_{1}, \alpha_{2}, \ldots\right) \in \prod$, perform a shift

$$
x \longmapsto x+\sum_{j \geq 1} \frac{\alpha_{j}}{K^{j N_{0}}} .
$$

Clearly

$$
\begin{gathered}
M_{N N_{0}}^{*}\left(x+\sum_{j \geq 1} \frac{\alpha_{j}}{K^{j N_{0}}}\right) \\
=M_{N_{0}}^{*}\left(x+\sum_{j \geq 1} \frac{\alpha_{j}}{K^{j N_{0}}}\right) M_{N_{0}}^{*}\left(K^{N_{0}} x+\sum_{j \geq 2} \frac{\alpha_{j}}{K^{(j-1) N_{0}}}\right) \ldots \\
M_{N_{0}}^{*}\left(K^{(N-1) N_{0}} x+\sum_{j \geq N} \frac{\alpha_{j}}{K^{(j-N+1) N_{0}}}\right)
\end{gathered}
$$


and, fixing $x \in \mathbb{T}, w \in \mathbb{R}^{2},|w|=1$,

$$
\begin{aligned}
& \log \left\|M_{N N_{0}}^{*}\left(x+\sum_{j \geq 1} \frac{\alpha_{j}}{K^{j N_{0}}}\right) w\right\| \\
& =\log \left\|M_{N_{0}}^{*}\left(x+\sum_{j \geq 1} \frac{\alpha_{j}}{K^{j N_{0}}}\right) w_{1}\left(\alpha_{j} ; j \geq 2\right)\right\| \\
& \quad+\log \left\|M_{N_{0}}^{*}\left(x+\sum_{J \geq 2} \frac{\alpha_{j}}{K^{(j-1) N_{0}}}\right) w_{2}\left(\alpha_{j} ; j \geq 3\right)\right\| \\
& +\cdots+\log \left\|M_{N_{0}}^{*}\left(x+\sum_{j \geq N} \frac{\alpha_{j}}{K^{(j-N+1) N_{0}}}\right) w_{N}\left(\alpha_{j} ; j \geq N+1\right)\right\|,
\end{aligned}
$$

where $w_{1}, w_{2}, \ldots$ are unit vectors in $\mathbb{R}^{2}$.

Rewrite the sum $\varphi_{1}+\varphi_{2}+\cdots+\varphi_{N}$ in (4.6) as

$$
\begin{aligned}
& d_{1}+d_{2}+\ldots+d_{N} \\
& + \\
& \mathbb{E}\left[\varphi_{1} \mid \alpha_{1}\right]+\cdots+\mathbb{E}\left[\varphi_{N} \mid \alpha_{N}\right],
\end{aligned}
$$

where $\left\{d_{j}=\varphi_{j}-\mathbb{E}\left[\varphi_{j} \mid \alpha_{j}\right]\right\}$ is a martingale difference sequence wrt the filtration $\prod$ introduced above. Also

$$
\begin{aligned}
& \max _{x \in \mathbb{T},|w|=1} \underset{\mathbb{Z} / K^{N_{0} \mathbb{Z}}}{A v} \log \left\|M_{N_{0}}^{*}\left(x+\frac{\alpha}{K^{N_{0}}}\right) w\right\| \\
& \geq \mathbb{E}\left[\varphi_{j} \mid \alpha_{j}\right] \\
& \geq \min _{x \in \mathbb{T},|w|=1} A v / K^{N_{0} \mathbb{Z}} \log \left\|M_{N_{0}}^{*}\left(x+\frac{\alpha}{K^{N_{0}}}\right) w\right\| .
\end{aligned}
$$

If we fix $N_{0}$ and take $K$ large enough, then, uniformly in $x$ and $w$ and with $f$ as in Theorem 1 ,

$$
\begin{aligned}
& \underset{\mathbb{Z} / K^{N_{0} \mathbb{Z}}}{A v} \log \left\|M_{N_{0}}^{*}\left(x+\frac{\alpha}{K^{N_{0}}}\right) w\right\| \\
& \approx \int_{\mathbb{T}^{N_{0}}} \log \left\|\left(\begin{array}{cc}
E-f\left(x_{0}\right) & 1 \\
-1 & 0
\end{array}\right) \ldots\left(\begin{array}{cc}
E-f\left(x_{N_{0}-1}\right) & 1 \\
-1 & 0
\end{array}\right) w\right\| d x_{0} \cdots d x_{N_{0}-1},
\end{aligned}
$$

as pointed out above, while, provided $N_{0}$ is chosen large enough, random matrix product theory implies that

$$
\frac{1}{N_{0}}(4.7) \asymp L(E)>0(=\text { Lyapunov exponent of the random cocycle }) \text {. }
$$


Hence, we get

$$
\left|L_{N N_{0}}\left(E ; x+\sum_{j \geq 1} \frac{\alpha_{j}}{K^{j N_{0}}}\right)-L(E)\right|<o(1)+\frac{1}{N N_{0}}\left|\sum_{j=1}^{N} d_{j}\right| .
$$

Applying the large deviation estimate for martingale difference sequences in the variable $\left(\alpha_{j}\right) \in \prod$, it follows that

$$
\operatorname{mes}\left[\alpha ;\left|\sum_{j=1}^{N} d_{j}\right|>\delta N N_{0}\right]<e^{-\delta^{\prime} N N_{0}}
$$

for some $\delta^{\prime}=\delta^{\prime}(\delta)>0$. Therefore also

$$
\operatorname{mes}\left[x \in \mathbb{T} ;\left|L_{N N_{0}}(E ; x)-L(E)\right|>\delta\right]<e^{-\delta^{\prime} N_{0} N},
$$

proving (4.5).

Remark 1. Take $f=\lambda f_{0}$ with $f_{0}$ satisfying (4.3) and let $\lambda$ vary. Theorem 1 then applies in any fixed range $0<\lambda_{1} \leq|\lambda| \leq \lambda_{2}$ (with a same $K$ ). For large $|\lambda|$ one easily derives positivity of (4.1) already for $N_{0}=2$. On the other hand, the small $\lambda$ case is captured by the Figolin-Pastur perturbative method (see [3], [2] ) provided $F$ is restricted to $[-2+\delta,-\delta] \cup[\delta, 2-\delta]$ for some $\delta>0$.

Remark 2. For given $f$, rather than deriving positivity of (4.1) for appropriately chosen large $N_{0}, K$ by invoking Furstenberg's theorem, one may of course proceed by a direct numerical verification at some scale $N_{0}$ and any given $K$. Hence positivity of the Lyapunov exponent for the model $x \mapsto K x$ with given $f$ may in principle be established numerically. We will illustrate this with some examples at the end of the paper.

\section{Toral automorphisms}

Let $\Omega=\mathbb{T}^{2}$ and $A=\left(\begin{array}{ll}a & b \\ c & d\end{array}\right) \in \mathrm{SL}_{2}(\mathbb{Z})$ acting on $\mathbb{T}^{2}$ (the approach works similarly in the higher dimensional case).

We assume $A$ strongly expanding with a large expanding eigenvalue

$$
K=\lambda_{+} \sim t=a+d
$$

and expanding eigenvector

$$
v=v_{+} \approx \frac{(a, c)}{V \overline{a^{2}+c^{2}}} .
$$

Assume also $\|A\| \sim K$. 
Returning to the discussion in Section 2, we apply the first scheme taking for

$$
\Omega_{N_{0}, N}=\left\{K^{-(N-1) N_{0}+\frac{1}{2}} t v ; 0 \leq t \leq 1\right\} .
$$

Thus (2.2) becomes

$$
\log \left\|M_{N_{0}}\left(A^{(N-1) N_{0}} x+t K^{\frac{1}{2}} v\right)\left(w_{x}\right)\right\|
$$

with

$$
w_{x}=\frac{M_{(N-1) N}(x) v}{\left\|M_{(N-1) N_{0}}(x) v\right\|} .
$$

In the present situation, the error terms (2.5) do not vanish and will be evaluated using the estimates from Section 3.

Clearly

$$
\left\|M_{N_{0}}\right\|,\left\|M_{N_{0}}^{-1}\right\| \leq\left(\|f\|_{\infty}+E+2\right)^{N_{0}}=C_{2}^{N_{0}}=C_{1}
$$

and

$$
\begin{aligned}
\left\|M_{N_{0}}\left(x+A^{\ell} y\right)-M_{N_{0}}(x)\right\| & \leq 2 C_{1}\left\|f^{\prime}\right\|_{\infty} K^{N_{0}+\ell-1}|y| \\
& <2 C_{1}\left\|f^{\prime}\right\|_{\infty} K^{-(N-2) N_{0}+\ell-\frac{1}{2}} .
\end{aligned}
$$

For $j=1, \ldots, N$, define

$$
A_{j}=M_{N_{0}}\left(T^{(N-j) N_{0}}(x+y)\right)=M_{N_{0}}\left(A^{(N-j) N_{0}} x+A^{(N-j) N_{0}} y\right)
$$

and

$$
B_{j}=M_{N_{0}}\left(T^{(N-j) N_{0}} x\right)=M_{N_{0}}\left(A^{N-j) N_{0}} x\right) .
$$

Applying (5.4) with $\ell=(N-j) N_{0}$ implies

$$
\left\|A_{j}-B_{j}\right\|<2 C_{1}\left\|f^{\prime}\right\|_{\infty} K^{-(j-2) N_{0}-\frac{1}{2}}=\varepsilon_{j} .
$$

Condition (3.10) becomes

$$
2 C_{1}^{3}\left\|f^{\prime}\right\|_{\infty} \sum_{j \geq 2} K^{-(j-2) N_{0}-\frac{1}{2}}\left(1+C_{1}\right)^{2 j-1}<\frac{1}{5},
$$

which by (5.3) will be satisfied if

$$
K>10^{3} C_{1}^{6}\left(1+C_{1}\right)^{6}\left(1+\left\|f^{\prime}\right\|_{\infty}\right)^{2} .
$$

An application of (3.11) gives then

$$
|(2.3)|,|(2.5)|<6 C_{1}^{2} \sum_{j \geq 2} j \varepsilon_{j}\left(1+C_{1}\right)^{2 j-1}<20 C_{1}^{3}\left(1+C_{1}\right)^{3}\left\|f^{\prime}\right\|_{\infty} K^{-\frac{1}{2}},
$$

which can be made arbitrarily small by choosing $K$ large. 
It remains to consider the image measure under the map (2.7)

$$
\begin{aligned}
\varphi:[0,1] \longrightarrow & \left(\mathrm{T}^{2}\right)^{N_{0}}, \\
t \longmapsto & \left(A^{(N-1) N_{0} x+K^{\frac{1}{2}} t v,}\right. \\
& A^{(N-1) N_{0}+1} x+K^{\frac{3}{2}} t v, \\
& \cdots, \\
& \left.A^{N N_{0}-1} x+K^{N_{0}-\frac{1}{2}} t v\right),
\end{aligned}
$$

or, equivalently

$$
\begin{aligned}
\psi:[0,1] & \longrightarrow\left(\mathrm{T}^{2}\right)^{N_{0}}, \\
t & \longmapsto\left(K^{\frac{1}{2}} t v, K^{\frac{3}{2}} t v, \ldots, K^{N_{0}-\frac{1}{2}} t v\right) .
\end{aligned}
$$

Estimating the Fourier transform of the image measure $\eta$ of $\psi$ on $\left(\mathrm{T}^{2}\right)^{N_{0}}$, we get for $\left(\xi_{0}, \ldots, \xi_{N_{0}-1}\right) \in\left(\mathbb{Z}^{2}\right)^{N_{0}}$

$$
\begin{aligned}
\left|\hat{\eta}\left(\xi_{0}, \ldots, \xi_{N_{0-1}}\right)\right| & =\left|\int_{0}^{1} e\left(K^{\frac{1}{2}}\left(v \cdot \xi_{0}+K v \cdot \xi_{1}+\cdots+K^{N_{0}-1} v \cdot \xi_{N_{0}-1}\right) t\right) d t\right| \\
& <4\left[1+K^{\frac{1}{2}}\left|v \cdot \xi_{0}+K v \cdot \xi_{1}+\cdots+K^{N_{0}-1} v \cdot \xi_{N_{0}-1}\right|\right]^{-1} .
\end{aligned}
$$

We restrict ourselves to frequencies $\left(\xi_{0}, \ldots, \xi_{N_{0}-1}\right) \in\left(\mathbb{Z}^{2}\right)^{N_{0}}$ with

$$
\left|\xi_{j}\right|<B=B_{f}
$$

We assume $A$ sufficiently mixing, in the sense that the expanding vector $v$ satisfies a Diophantine property

$$
|\langle v, \xi\rangle|>\frac{1}{B_{1}}, \quad \text { for all } \xi \in \mathbb{Z}^{2} \backslash\{0\},|\xi|<B .
$$

Also assume $K=\lambda_{+}>2 B B_{1}$. It follows then from (5.9) that

$$
(5.8)<\frac{4 B_{1}}{K^{\frac{1}{2}}}
$$

if $\left(\xi_{0}, \ldots, \xi_{N_{0}-1}\right) \in\left(\mathbb{Z}^{2}\right)^{N_{0}} \backslash\{0\},\left|\xi_{j}\right|<B$. We obtain the following result.

Theorem 2. Consider the Schrodinger operator on $\mathbb{Z}$

$$
H_{x}=\Delta+\lambda V_{x}, \quad \text { with } V_{x}(j)=f\left(A^{j} x\right),
$$

with $f$ a non-constant function in $C^{1}\left(\mathrm{~T}^{2}\right), A \in \mathrm{SL}_{2}(\mathbb{Z})$. Assuming A sufficiently mixing (depending on $\operatorname{osc}\left(\left.f\right|_{\mathbb{T}^{2}}\right)$ and $\|f\|_{C^{1}}$ ), we obtain positive Lyapunov exponents for all energies, Hölder regularity of the IDS and Anderson localization. 


\section{Further comments and numerical aspects}

As pointed out already, the required positivity of (2.6) at some scale $N_{0}$ may be verifiable numerically, short of a theoretical reason, and in this way extensions of Theorems 1 and 2 could be obtained in situations where a former transference to a random setting is not possible. Observe also that in our problem, one may replace $M_{N}(x)$ by $S M(x) S^{-1}$ for any chosen similarity $S \in \mathrm{SL}_{2}(\mathrm{R})$ such that $\|S\|<C$ independently from $N$. Therefore, instead of establishing positivity of (2.6), we can as well consider

$$
\min _{N} \min _{x \in \Omega,|w|=1} A v\left\{\log \left\|S M_{N_{0}}\left(T^{(N-1) N_{0}}(x+y)\right) S^{-1} w\right\|\right\}
$$

for a fixed $S \in \mathrm{GL}_{2}(\mathbb{R})$. In particular, using the Figolin-Pastur formalism, one may represent the Schrödinger matrices in polar coordinates (which is especially useful in the small $\lambda$ regime).

Fix $\delta>0$ and assume

$$
|E|<2-\delta \text {. }
$$

Denoting $v_{n}=f\left(T^{n} x\right)$, define $\kappa \in(0, \pi)$ and $V_{n}$ by

$$
\begin{gathered}
E=2 \cos \kappa, \\
V_{n}=-\frac{v_{n}}{\sin \kappa},
\end{gathered}
$$

and let

$$
S=\left(\begin{array}{cc}
1 & -\cos \kappa \\
0 & \sin \kappa
\end{array}\right)
$$

Then

$$
M_{N}=\prod_{n=N-1}^{0}\left(\begin{array}{cc}
E-\lambda v_{n} & -1 \\
1 & 0
\end{array}\right)
$$

is converted to

$$
M_{N}^{\prime}=\prod_{n=N-1}^{0}\left[\left(\begin{array}{cc}
\cos \kappa & -\sin \kappa \\
\sin \kappa & \cos \kappa
\end{array}\right)+\lambda V_{n}\left(\begin{array}{cc}
\sin \kappa & \cos \kappa \\
0 & 0
\end{array}\right)\right] .
$$

One should also expect that for small $\lambda$, the representation (6.6) is more suited for numerics as the factors are perturbations of a rotation. 
As an example related to $\S 4$ of such numerics, we considered the map

$$
x \longmapsto 2 x \quad(K=2),
$$

and evaluated (4.1) in the energy range $[-\sqrt{2}, \sqrt{2}]$ for different couplings $\lambda$ and using the transformation (6.6). It turns out that $N_{0}=6$ already suffices to obtain the positivity for $|\lambda| \geq \frac{2}{5}$.

In the displays below, we dropped the irrelevant factor $K^{-N_{0}}$ in (4.1).

Further numerical work on the positivity of the Lyapunov exponent using our method will appear in the arXiv version of the paper.

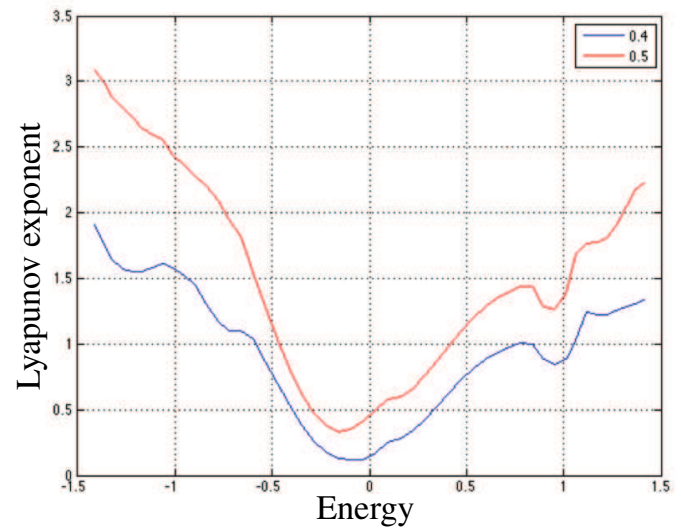

Figure 1. Positivity of Lyapunov Exponents for $\lambda=0.4$ and $\lambda=0.5$.

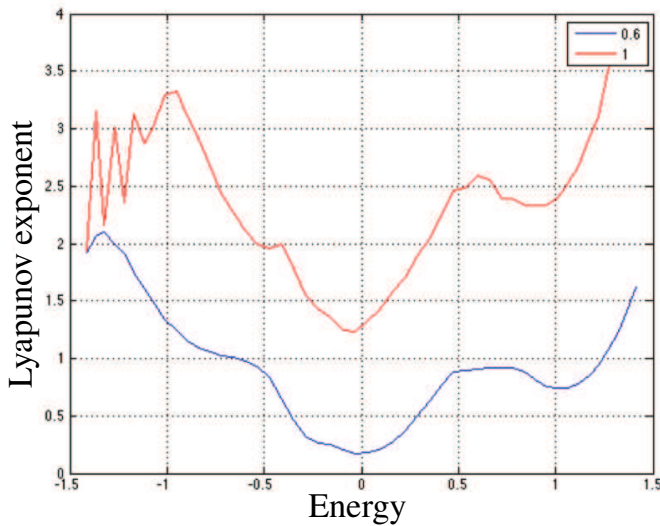

Figure 2. Positivity of Lyapunov Exponents for $\lambda=0.6$ and $\lambda=1$. 


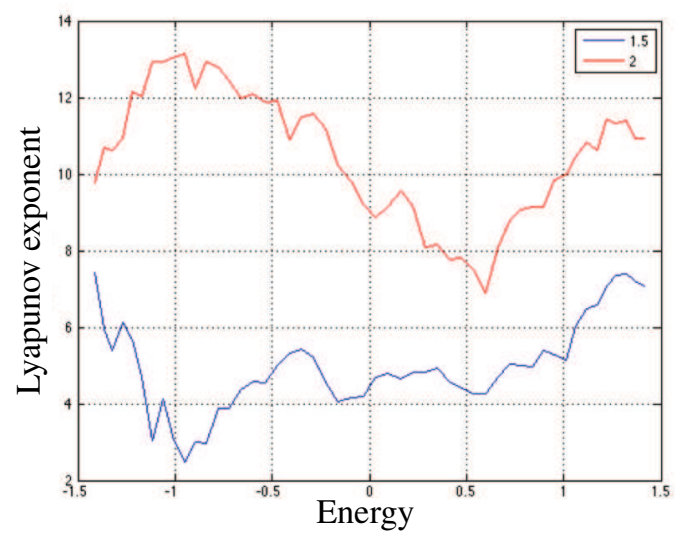

Figure 3. Positivity of Lyapunov Exponents for $\lambda=1.5$ and $\lambda=2$.

\section{References}

[1] A. Bougerol and J. Lacroix, Products of random matrices with applications to Schrödinger operators. Progress in Probability and Statistics, 8. Birkhäuser Boston, Boston, MA, 1985. MR 0886674 Zbl 0572.60001

[2] J. Bourgain and W. Schlag, Anderson localization for Schrödinger operators on $\mathbb{Z}$ with strongly mixing potentials. Comm. Math. Phys. 215 (2000), no. 1, 143-175. MR $1800921 \mathrm{Zbl} 1040.81014$

[3] V. Chulaevsky and T. Spencer, Positive Lyapunov exponents for a class of deterministic potentials. Comm. Math. Phys. 168 (1995), no. 3, 455-466. MR 1328248 Zbl 0821.60070

Received August 17, 2013; revised September 9, 2013

Jean Bourgain, Institute for Advanced Study, 1 Einstein Drive, Princeton, N.J. 08540, U.S.A.

e-mail: bourgain@math.ias.edu

Eric Bourgain-Chang, Department of Mathematics, University of California, 970 Evans Hall \#3840, Berkeley, CA 94720-3840, U.S.A.

e-mail: ebc@berkeley.edu 